\title{
Digraph representations of 2-closed permutation groups with a normal regular cyclic subgroup
}

\author{
Jing $\mathrm{Xu}^{*}$ \\ Department of Mathematics \\ Capital Normal University \\ Beijing 100048, China \\ xujing@cnu.edu.cn
}

Submitted: Mar 30, 2015; Accepted: Nov 13, 2015; Published: Nov 27, 2015

Mathematics Subject Classifications: 05C25, 20B25

\begin{abstract}
In this paper, we classify 2-closed (in Wielandt's sense) permutation groups which contain a normal regular cyclic subgroup and prove that for each such group $G$, there exists a circulant $\Gamma$ such that $\operatorname{Aut}(\Gamma)=G$.
\end{abstract}

\section{Introduction}

In 1969, Wielandt [15] introduced the concept of the 2-closure of a permutation group. Let $G$ be a finite permutation group on a set $\Omega$, the 2 -closure $G^{(2)}$ of $G$ on $\Omega$ is the largest subgroup of $\operatorname{Sym}(\Omega)$ containing $G$ that has the same orbits as $G$ in the induced action on $\Omega \times \Omega$, and we say $G$ is 2 -closed if $G=G^{(2)}$. It seems impossible to classify all 2-closed transitive permutation groups. However, certain classes of 2-closed transitive groups have been determined. For example, in $[16,17]$ the author determined all 2-closed odd-order transitive permutation groups of degree $p q$ where $p, q$ are distinct odd primes. In this paper, one of our main purposes is to classify all 2-closed permutation groups with a normal regular cyclic subgroup, see Theorem 1.2. Recall that a permutation group is regular if it is transitive and the only element that fixes a point is the identity. And for more information about the 2-closures of permutation groups containing a cyclic regular subgroup, see also [7].

Another research topic of this paper is the study of the automorphism groups of (di)graphs. The full automorphism group of a (di)graph $\Gamma$ must be 2-closed since any permutation of the vertex set that preserves the orbits of $\operatorname{Aut}(\Gamma)$ on ordered pairs preserves adjacency. However, not every 2-closed permutation group is the full automorphism group

*This work was supported by NSFC (project number 10901110, 11371259). 
of some (di)graph. Therefore, the concept of 2-closed groups is more general than the concept of the full automorphism groups of (di)graphs, and the classification of 2-closed groups is closely related to the study of the full automorphism groups of the corresponding digraphs. In this paper, in order to determine 2-closed groups that contain a normal regular cyclic subgroup, we also study circulant digraphs, that is Cayley digraphs of cyclic groups. See Section 2 for a more detailed explanation.

Furthermore, we discuss the following representation problem. A digraph $\Gamma$ with vertex set $\Omega$ is said to represent a permutation $\operatorname{group} G \leqslant \operatorname{Sym}(\Omega)$ if $\operatorname{Aut}(\Gamma)=G$. In this case, we also say that the permutation group $G$ has a digraph representation $\Gamma$.

Digraph representation problem: given a 2-closed group $G$, is there a digraph $\Gamma$ that represents $G$ ?

Suppose the digraph $\Gamma$ represents a 2 -closed group $G \leqslant \operatorname{Sym}(\Omega)$. Then for any $g \in$ $\operatorname{Sym}(\Omega)$, to determine whether $g$ lies in $G$ we only need to test if $g$ preserves the single 2 -relation given by the arc set of $\Gamma$, instead of checking all $G$-invariant 2 -relations. We say a digraph $\Gamma$ is arc-transitive if $\operatorname{Aut}(\Gamma)$ is transitive on the arc set of $\Gamma$. This means, the arc set of $\Gamma$ is actually a minimal Aut $(\Gamma)$-invariant 2-relation. Suppose further that the 2-closed group $G$ can be represented by an arc-transitive digraph $\Gamma$. Then a permutation $g$ lies in $G$ if and only if $g$ leaves invariant the minimal $G$-invariant 2-relation given by the arc set of $\Gamma$. We will show that there are arc-transitive digraph representations for most 2-closed groups that contain a normal regular cyclic subgroup, see the remark after Lemma 3.12.

Replacing digraph with graph, we obtain the graph representation problem which asks for an undirected graph to represent a 2-closed group. These two questions have previously appeared in the literature, see for example $[1,4]$. Clearly, the graph version problem is much more complicated than the digraph one. Since we are interested in understanding the concept of 2-closed groups, we concentrate on the digraph representation problem in this paper.

A regular permutation group is 2-closed, and in 1980, Babai [2] proved that with five exceptions, every finite regular permutation group occurs as the automorphism group of a digraph. This is the famous DRR (digraphical regular representations) problem [2]. It is proved in [14] that for any prime power $q$, the semilinear group $\Gamma L(1, q)$ can be represented by an arc-transitive circulant digraph. Moreover, it is shown in $[16,17]$ that every 2closed odd-order transitive permutation group of degree $p q$ has a tournament digraph representation. As for graphical representation problem, see for example [3, 6, 8, 9, 10, 13].

In this paper, we will prove that every 2-closed permutation group $G$ with a normal regular cyclic subgroup is the full automorphism group of a circulant digraph. We may suppose that $G=Z_{n} \rtimes G_{0}$ acting on $Z_{n}$ naturally where $G_{0} \leqslant \operatorname{Aut}\left(Z_{n}\right)$. We first describe the necessary and sufficient condition for $G_{0}$ such that $G$ is 2-closed. For the detailed explanation of notation, see Section 2 and Section 3.3.1.

Conditions 1.1. Let $n=2^{d_{1}} p_{2}^{d_{2}} \cdots p_{t}^{d_{t}}, d_{1} \geqslant 0, d_{2}, \ldots, d_{t} \geqslant 1, t \geqslant 1$ where $p_{2}, \ldots, p_{t}$ are distinct odd primes (also write $p_{1}=2$ ). And let $\operatorname{Aut}\left(Z_{n}\right)=\operatorname{Aut}\left(Z_{2^{d_{1}}}\right) \times \cdots \times \operatorname{Aut}\left(Z_{p_{t}^{d_{t}}}\right)=$ $D_{1} D_{2} \cdots D_{t}$, where $D_{i}$ is the direct factor subgroup of $\operatorname{Aut}\left(Z_{n}\right)$ that fixes each component 
of the elements of $Z_{n}$ except for the $i$-th component. So $D_{i} \cong \operatorname{Aut}\left(Z_{p_{i}}\right)$ for each $i$. In fact $D_{i}$ induces a faithful action on the subgroup $Z_{p_{i}}{ }_{i}$. Note that the induced action $D_{1}$ on the subgroup $Z_{2^{d_{1}}}$ is permutation isomorphic to $\left\langle(-1)^{*}\right\rangle \times\left\langle 5^{*}\right\rangle\left(d_{1} \geqslant 3\right)$, the multiplicative group of units of the ring $\mathbb{Z}_{2^{d_{1}}}$ acting on the additive group $\mathbb{Z}_{2^{d_{1}}}$, let $\phi:\left\langle(-1)^{*}\right\rangle \times\left\langle(5)^{*}\right\rangle \rightarrow D_{1}$ be the corresponding group isomorphism.

Let $G_{0} \leqslant \operatorname{Aut}\left(Z_{n}\right)$.

(i) if $i \geqslant 2, d_{i}=1$ and $p_{i} \geqslant 5$, then $D_{i} \nless G_{0}$.

(ii) if $i \geqslant 2$ and $d_{i} \geqslant 2$, then $D_{i} \cap G_{0} \leqslant Z_{p_{i}-1}$.

(iii) if $d_{1}=3$, then $D_{1} \nless G_{0}$.

(iv) if $d_{1} \geqslant 4$, then either $\left|D_{1} \cap G_{0}\right| \leqslant 2$ or $\left|D_{1} \cap G_{0}\right|=4$ and $D_{1} \cap G_{0} \nless\left\langle\phi\left(5^{*}\right)\right\rangle$.

The main result of this paper is the following theorem.

Theorem 1.2. Suppose $G=Z_{n} \rtimes G_{0}$ acting on $Z_{n}$ naturally where $G_{0} \leqslant \operatorname{Aut}\left(Z_{n}\right)$. Then $G$ is 2-closed if and only if $G_{0}$ satisfies Conditions 1.1. Moreover, if $G$ is 2-closed then $G$ can be represented by a circulant digraph.

\section{Preliminary results and notation}

First we introduce some concepts and notation concerning Cayley digraphs. Given a finite group $H$, and a subset $S \subset H \backslash\{1\}$, the Cayley digraph $\Gamma=\operatorname{Cay}(H, S)$ with respect to $S$ is defined as the directed graph with vertex set $H$ and $\operatorname{arc}$ set $A \Gamma=\{(g, s g) \mid g \in H, s \in S\}$. Moreover, a Cayley digraph of a cyclic group is called a circulant. It is easy to check that the right regular representation $\hat{H}$ is contained in $\operatorname{Aut}(\Gamma)$. In fact, a digraph is a Cayley digraph if and only if its automorphism group contains a regular subgroup. Moreover let $\operatorname{Aut}(H, S)=\left\{\sigma \in \operatorname{Aut}(H) \mid S^{\sigma}=S\right\}$, then each element in $\operatorname{Aut}(H, S)$ induces an automorphism of the Cayley digraph $\Gamma=\operatorname{Cay}(H, S)$. It is proved in [10] that the normalizer of $\hat{H}$ in $\operatorname{Aut}(\Gamma)$ is $\hat{H} \rtimes \operatorname{Aut}(H, S)$. We say a Cayley digraph $\Gamma=\operatorname{Cay}(H, S)$ is normal if $\hat{H}$ is normal in $\operatorname{Aut}(\Gamma)$, that is, $\operatorname{Aut}(\Gamma)=\hat{H} \rtimes \operatorname{Aut}(H, S)$, see [10, 18]. So the automorphism group of a normal circulant must be a 2-closed group that contains a normal regular cyclic group. Conversely, we will show that each such 2-closed group is the automorphism group of some normal circulant.

Throughout the rest of this paper, let $Z_{n}$ be an abstract cyclic group of order $n$ and let $G \leqslant \operatorname{Sym}\left(Z_{n}\right)$ be a transitive permutation group which contains a normal regular cyclic group $\hat{Z}_{n}$ where

$$
\hat{Z}_{n}=\left\{\hat{g}: x \rightarrow x g \forall x \in Z_{n} \mid g \in Z_{n}\right\} .
$$

Therefore $G$ is a semidirect product $\hat{Z}_{n} \rtimes G_{0}$ for some subgroup $G_{0} \leqslant \operatorname{Aut}\left(Z_{n}\right)$ acting naturally on $Z_{n}$. Since $\hat{Z}_{n} \cong Z_{n}$, we may also write $G=Z_{n} \rtimes G_{0}$ directly. Our goal is to determine all such 2-closed groups. 
The mail tool used in this paper is the Kovács-Li classification of arc-transitive circulants [11, 12]. Praeger and the author [14] refined the Kovács-Li classification and obtained the following theorem.

Theorem 2.1. [14, Theorem 1.1] Let $G=Z_{n} \rtimes G_{0} \leqslant Z_{n} \rtimes \operatorname{Aut}\left(Z_{n}\right)$ acting naturally on $Z_{n}$. Then, up to isomorphism, there is a unique connected $Z_{n}$-circulant $\Gamma$ on which $G$ acts arc-transitively. Moreover either $\operatorname{Aut}(\Gamma)=G$ or one of the following holds.

(a) $n=p \geqslant 5$ is prime, $\Gamma=K_{p}$, and $G=\operatorname{AGL}(1, p)$;

(b) $n=b m>4$, where $b \geqslant 2$, $p$ divides $m$ for each prime $p$ dividing $b, \Gamma=\Sigma\left[\bar{K}_{b}\right]$;

(c) $n=p m$, where $p$ is prime, $5 \leqslant p<n$, and $\operatorname{gcd}(m, p)=1, \Gamma=\Sigma\left[\bar{K}_{p}\right]-p \cdot \Sigma$, $G_{0}=\operatorname{Aut}\left(Z_{p}\right) \times H \leqslant \operatorname{Aut}\left(Z_{p}\right) \times \operatorname{Aut}\left(Z_{m}\right)$, and $\Sigma$ is a connected $\left(Z_{m} \rtimes H\right)$-arctransitive $Z_{m}$-circulant.

We point out that up to isomorphism, in the above theorem $\Gamma$ can be defined as $\operatorname{Cay}\left(Z_{n}, z^{G_{0}}\right)$ where $z$ is a generator of $Z_{n}$ and $z^{G_{0}}$ is the orbit of $z$ under $G_{0}$. Moreover, if case (b) happens, then the group $Z_{n}$ has a subgroup $Y$ of order $b$, and $\Gamma=\operatorname{Cay}\left(Z_{n}, S\right)$ where $S$ is a union of $Y$-cosets each consisting of generators for $Z$.

As a simple application of Theorem 2.1, we determine the 2-closed transitive permutation groups of degree $p$ where $p$ is a prime.

Corollary 2.2. Let $p$ be a prime. Let $G \leqslant \operatorname{Sym}(\Omega)$ be a 2-closed transitive permutation group of degree $p$. Then there exists a digraph representing $G$. Moreover, $G$ is one of the following.

1. The symmetric group $S_{p}(p \geqslant 2)$ which is 2-transitive on $\Omega$.

2. An affine subgroup $Z_{p} \rtimes Z_{k}$ where $p \geqslant 3,1 \leqslant k<(p-1)$ and $k \mid(p-1)$.

Conversely, each group of the above two types is 2-closed.

Proof. Suppose $G$ is a 2-closed transitive permutation group of degree $p$. By a classical result of Burnside, $G$ is either 2-transitive or is affine. If $G$ is 2-transitive, then $G=G^{(2)}=$ $S_{p}$ and $p \geqslant 2$. If $G$ is not 2 -transitive, then $G=Z_{p} \rtimes Z_{k}$ where $p \geqslant 3,1 \leqslant k<(p-1)$ and $k \mid(p-1)$.

For the converse, note that $S_{p}$ is the full automorphism group of the complete graph $K_{p}$ and so $S_{p}$ is indeed 2-closed. Next, let $G=Z_{p} \rtimes Z_{k}$ where $p \geqslant 3,1 \leqslant k<(p-1)$ and $k \mid(p-1)$. By Theorem 2.1, there is a connected arc-transitive circulant $\Gamma$ of order $p$ such that $\operatorname{Aut}(\Gamma)=G$, and so $G$ is 2 -closed.

Remark: If $p=2,3$ then $S_{p}=Z_{p} \rtimes \operatorname{Aut}\left(Z_{p}\right)$ is 2-closed; and if $p \geqslant 5$ then $Z_{p} \rtimes \operatorname{Aut}\left(Z_{p}\right)$ is not 2-closed.

We also need the following theorem.

Theorem 2.3. [5, Theorem 5.1] Let $G_{1} \leqslant \operatorname{Sym}\left(\Omega_{1}\right)$ and $G_{2} \leqslant \operatorname{Sym}\left(\Omega_{2}\right)$ be transitive permutation groups. Consider the natural product action of $G_{1} \times G_{2}$ on $\Omega_{1} \times \Omega_{2}$. Then $\left(G_{1} \times G_{2}\right)^{(2)}=G_{1}^{(2)} \times G_{2}^{(2)}$. 
Finally, we fix the following notation. Let $A \leqslant \operatorname{Sym}(\Omega)$. Suppose that $A_{B}$ is the setwise stabilizer of $B \subseteq \Omega$ and $g \in A_{B}$, we denote $A_{B}^{B}$ to be the induced permutation group on $B$ by $A_{B}$ and denote $g^{B}$ to be the induced permutation on $B$ by $g$.

\section{2-closed groups containing a normal regular cyclic group}

In this section we classify 2-closed groups $G$ that contain a normal regular cyclic group $Z_{n}$. With notation in Section 2, we may suppose that $G=Z_{n} \rtimes G_{0} \leqslant Z_{n} \rtimes \operatorname{Aut}\left(Z_{n}\right)$ acting naturally on $Z_{n}$. We first handle the special case that $n$ is a prime power in Subsection 3.1 and Subsection 3.2. The notation needed for the statement of Theorem 1.2 is given in Subsection 3.3.1 and the proof is given in Subsection 3.3.2.

\subsection{The case $n=p^{d}$ with $p$ an odd prime}

Let $n=p^{d}$ where $p$ is an odd prime and $d \geqslant 2$ is an integer. Then $\operatorname{Aut}\left(Z_{n}\right)=Z_{(p-1)} \times Z_{p^{d-1}}$ is a cyclic group. We take $\alpha \in \operatorname{Aut}\left(Z_{n}\right)$ such that $o(\alpha)=p$, then there exists $\gamma \in \operatorname{Aut}\left(Z_{n}\right)$ with order $p^{d-1}$ such that $\alpha=\gamma^{p^{d-2}}$. We first look at the action of $\alpha$ on $Z_{n}$.

Let $H=Z_{p^{d-1}}$ be the unique subgroup of $Z_{n}$ of order $p^{d-1}$. Let $N=Z_{n} \rtimes \operatorname{Aut}\left(Z_{n}\right)$. Then the cosets of $H$ form a block system $\mathcal{B}$ of $N$ on $Z_{n}$. Denote $\mathcal{B}=\left\{B_{1}=H, B_{2}, \ldots, B_{p}\right\}$. Since the elements in $B_{2}, \ldots, B_{p}$ are of order $p^{d}, \gamma$ fixes each block setwise and $\gamma^{B_{i}}$ is a $p^{d-1}$-cycle for each $i \geqslant 2$. However, $\gamma$ fixes the point $1 \in H=B_{1}$, so the order of $\gamma^{B_{1}}$ is strictly less than $p^{d-1}$. It then follows that $\alpha$ fixes $B_{1}$ pointwise and is fixed point free on each $B_{i}$ for $i \geqslant 2$.

On the other hand, let $N_{B_{i}}^{B_{i}}$ be the induced permutation group of the setwise stabilizer $N_{B_{i}}$ on $B_{i}$. Then $N_{B_{i}}^{B_{i}}=\hat{Z}_{p^{d-1}} \rtimes K_{i}$ and $K_{i} \cong \operatorname{Aut}\left(Z_{p^{d-1}}\right),\left(\hat{Z}_{p^{d-1}}\right.$ is defined in equation (1)). For each $i \geqslant 2$, since $\gamma^{B_{i}}$ is fixed point free, we have that $\gamma^{B_{i}}=\hat{y}_{i}^{B_{i}} \tau$ where $1 \neq y_{i} \in$ $H \leqslant Z_{n}$ and $\tau \in K_{i}$. Since $\tau$ normalizes $\hat{Z}_{p^{d-1}},\left(\gamma^{B_{i}}\right)^{2}=\hat{y}_{i}^{B_{i}}\left(\tau \hat{y}_{i}^{B_{i}} \tau^{-1}\right) \tau \tau=a_{i 2} \tau^{2}$ where $a_{i 2}$ is some element in $\hat{Z}_{p^{d-1}}$. By induction, we have that for each $k \geqslant 1,\left(\gamma^{B_{i}}\right)^{k}=a_{i k} \tau^{k}$ where $a_{i k}$ is some element in $\hat{Z}_{p^{d-1}}$. Since $\gamma^{B_{i}}$ is of order $p^{d-1}$ and $\hat{Z}_{p^{d-1}} \cap K_{i}=\{1\}$, we have that $\tau^{p^{d-1}}=1$. Since $\tau \in \operatorname{Aut}\left(Z_{p^{d-1}}\right)=Z_{p-1} \times Z_{p^{d-2}}, \tau^{p^{d-2}}=1$. Recall that $\alpha=\gamma^{p^{d-2}}$, it then follows that $\alpha^{B_{i}}$ is $\hat{x}_{i}^{B_{i}}$ for some $x_{i} \in Z_{n}$ with order $p$. Note that $x_{i}$ may not equal $x_{j}$ for $2 \leqslant i<j \leqslant p$, but they are all of order $p$. We have proved the following lemma.

Lemma 3.1. Let $\alpha \in \operatorname{Aut}\left(Z_{p^{d}}\right)$ with order $p$. Let $\mathcal{B}=\left\{B_{1}=H, B_{2}, \ldots, B_{p}\right\}$ be the cosets of the subgroup $H$ where $H<Z_{p^{d}}$ is of order $p^{d-1}$. Then $\alpha$ fixes $B_{1}=H$ pointwise and for each $i \geqslant 2, \alpha^{B_{i}}$ is $\hat{x}_{i}^{B_{i}}$ for some $x_{i} \in Z_{n}$ with order $p$.

Corollary 3.2. Let $n=p^{d}$ and $Z_{n}=\langle z\rangle$. Let $Z_{p} \leqslant Z_{n}$ be the subgroup of order $p$. Suppose that $G=Z_{n} \rtimes G_{0}$ where $G_{0} \leqslant \operatorname{Aut}\left(Z_{n}\right)$. Then the coset $z Z_{p} \subseteq z^{G_{0}}$ if and only if $p \| G_{0} \mid$.

Remark: Let $S=z^{G_{0}}$ and $\Gamma=\operatorname{Cay}\left(Z_{n}, S\right)$. If case (b) of Theorem 2.1 occurs for $\Gamma$, then $z Z_{p} \subseteq z^{G_{0}}$. That is why we consider this corollary. 
Proof. Let $\operatorname{Aut}\left(Z_{p^{d}}\right)=\langle\mu\rangle \times\langle\gamma\rangle=Z_{p-1} \times Z_{p^{d-1}}$ and $\alpha=\gamma^{p^{d-2}}$. Then $p \| G_{0} \mid$ if and only if $\alpha \in G_{0}$.

Let $\mathcal{B}=\left\{B_{1}=H, B_{2}, \ldots, B_{p}\right\}$ be the cosets of the subgroup $H$ where $H<Z_{p^{d}}$ is of order $p^{d-1}$. Then it is easy to show that $\mu$ fixes $B_{1}$ setwise, and permutes $B_{2}, \ldots, B_{p}$ as a $(p-1)$-cycle.

By Lemma 3.1, if $\alpha \in G_{0}$ then $z Z_{p} \subseteq z^{G_{0}}$. Conversely, suppose that $z Z_{p} \subseteq z^{G_{0}}$. Note that the generator $z \in B_{k}$ for some $k \geqslant 2$ and $z Z_{p} \subseteq B_{k}$. By the action of $\mu$ and $\gamma$, we conclude that $\alpha \in G_{0}$.

Proposition 3.3. Let $n=p^{d}$ where $p$ is an odd prime and $d \geqslant 2$. Let $G=Z_{n} \rtimes G_{0} \leqslant$ $Z_{n} \rtimes \operatorname{Aut}\left(Z_{n}\right)$ acting naturally on $Z_{n}$. Then $G$ is 2-closed if and only if $G_{0} \leqslant Z_{p-1}$. Moreover, if $G$ is 2-closed then $G$ can be represented by an arc-transitive circulant.

Proof. As defined at the beginning of Subsection 3.1, let $\alpha \in \operatorname{Aut}\left(Z_{p^{d}}\right)$ be an element of order $p$. Let $\mathcal{B}=\left\{B_{1}=H, B_{2}, \ldots, B_{p}\right\}$ be the cosets of the subgroup $H$ where $H<Z_{p^{d}}$ is of order $p^{d-1}$.

Suppose first that $G_{0} \nless Z_{p-1}$, that is $p \| G_{0} \mid$, then $\alpha \in G_{0}$. By Lemma 3.1, $\alpha$ fixes $B_{1}=H$ pointwise and for each $i \geqslant 2, \alpha^{B_{i}}$ is $\hat{x}_{i}^{B_{i}}$ for some $x_{i} \in Z_{n}$ with order $p$.

Let $1 \neq \beta \in \operatorname{Sym}\left(Z_{n}\right)$ such that $\beta$ fixes every element of $B_{1}, \ldots, B_{p-1}$ and $\beta^{B_{p}}=\alpha^{B_{p}}$. That means $\beta^{B_{p}}=\hat{x}_{p}^{B_{p}}$, (recall that $\hat{x}: z \mapsto z x$ for any $z \in Z_{n}$ ). We claim that $\beta \in\left(Z_{p^{d}} \rtimes\langle\alpha\rangle\right)^{(2)}$ and so $\beta \in G^{(2)}$. Take any pair $\left(y_{1}, y_{2}\right) \in Z_{n} \times Z_{n}$. If both $y_{1}$ and $y_{2}$ belong to $B_{p}$, then $\left(y_{1}, y_{2}\right)^{\beta}=\left(y_{1} x_{p}, y_{2} x_{p}\right)$ is in the orbital $\left(y_{1}, y_{2}\right)^{G}$. Suppose next that exactly one of $\left\{y_{1}, y_{2}\right\}$ lies in $B_{p}$, say $y_{2} \in B_{p}$. Since the stabilizer $G_{y_{1}}$ is the conjugate of $G_{0}$ in $G$ by an element in $\hat{Z}_{n}$, a conjugate of $\alpha$, say $\rho$, is in $G_{y_{1}}$. Therefore $\beta^{B_{p}}$ equals $\left(\rho^{j}\right)^{B_{p}}$ for some $j \in\{1, \ldots, p-1\}$, and so $\left(y_{1}, y_{2}\right)^{\beta} \in\left(y_{1}, y_{2}\right)^{G}$. It then follows that $\beta \in\left(Z_{p^{d}} \rtimes\langle\alpha\rangle\right)^{(2)} \leqslant G^{(2)}$. However, since $\beta$ fixes $B_{1}$ and $B_{2}$ pointwise, $\beta \notin Z_{p^{d}} \rtimes \operatorname{Aut}\left(Z_{p^{d}}\right)$, and so $\beta \notin G$ and $G$ is not 2 -closed.

Suppose next that $G_{0} \leqslant Z_{p-1}$. Let $S=z^{G_{0}}$ where $z \in Z_{p^{d}}$ is an element of order $p^{d}$ and let $\Gamma=\operatorname{Cay}\left(Z_{n}, S\right)$. Since $\left(p,\left|G_{0}\right|\right)=1, p \nmid|S|$ and so $S$ is not a union of cosets of any subgroup of $Z_{n}$. By Theorem 2.1, $\operatorname{Aut}(\Gamma)=G$ and so $G$ is 2-closed. This completes the proof.

Remark: In above proof, note that $\beta$ is in $\left(Z_{p^{d}} \rtimes\langle\alpha\rangle\right)^{(2)}$. Hence we actually proved that $\left(Z_{p^{d}} \rtimes\langle\alpha\rangle\right)^{(2)} \not \leq Z_{p^{d}} \rtimes \operatorname{Aut}\left(Z_{p^{d}}\right)$ where $\alpha \in \operatorname{Aut}\left(Z_{p^{d}}\right)$ is of order $p$.

\subsection{The case $n=2^{d}$ for $d \geqslant 2$}

Notation: For convenience, in this subsection we write $Z_{n}$ additively as the group $\mathbb{Z}_{n}$ of integers modulo $n$, so in this case

$$
\hat{Z}_{n}=\hat{\mathbb{Z}}_{n}=\left\{\hat{x}: g \rightarrow g+x \mid x \in Z_{n}\right\} .
$$

Moreover $\operatorname{Aut}\left(Z_{n}\right)$ is the multiplicative group $\mathbb{Z}_{n}^{*}$ so that $i^{*} \in \operatorname{Aut}\left(Z_{n}\right)$ denotes the map $j \mapsto i j$. 


\subsection{1 $d=2$ :}

In this case, $\operatorname{Aut}\left(Z_{4}\right)=\left\langle(-1)^{*}\right\rangle \cong Z_{2}$. We have the following result.

Lemma 3.4. Suppose that $\hat{Z}_{4} \leqslant G \leqslant \hat{Z}_{4} \rtimes\left\langle(-1)^{*}\right\rangle \cong D_{8}$. Then $G$ is 2-closed and is the full automorphism group of an arc-transitive circulant.

Proof. Either $G \cong Z_{4}$ is regular or $G \cong D_{8}$. Note that $\operatorname{Aut}\left(\operatorname{Cay}\left(\mathbb{Z}_{4},\{1\}\right)\right)=Z_{4}$ and $\operatorname{Aut}\left(\operatorname{Cay}\left(\mathbb{Z}_{4},\{1,-1\}\right)\right)=D_{8}=Z_{4} \rtimes Z_{2}$, this proves the lemma.

Remark: By [14, Lemma 2.3], a connected arc-transitive circulant $\Gamma$ is both normal and of lexicographic product form if and only if $\Gamma=\operatorname{Cay}\left(\mathbb{Z}_{4},\{1,-1\}\right)$ and $\operatorname{Aut}(\Gamma)=$ $Z_{4} \rtimes \operatorname{Aut}\left(Z_{4}\right)$. In this case the orbit $1^{\operatorname{Aut}\left(\mathbb{Z}_{4}\right)}=\{1,3\}=1+Z_{2}$ is a coset of $Z_{2}$.

\subsection{2 $\quad d \geqslant 3:$}

In this case, $\operatorname{Aut}\left(Z_{n}\right)=\left\langle(-1)^{*}\right\rangle \times\left\langle 5^{*}\right\rangle \cong Z_{2} \times Z_{2^{d-2}}$. Denote $N=\hat{\mathbb{Z}}_{n} \rtimes \mathbb{Z}_{n}^{*}$. Let $H$ be the unique subgroup of $\mathbb{Z}_{n}$ with order $2^{d-2}$. Let $B_{0}=H, B_{1}=1+H, B_{2}=2+H, B_{3}=3+H$ be the cosets of $H$, then $\mathcal{B}=\left\{B_{0}, B_{1}, B_{2}, B_{3}\right\}$ forms a complete block system of $N$ on $\mathbb{Z}_{n}$.

We first study the action of $5^{*}$. By computation $5^{*}$ preserves each block $B_{i}$, we determine the induced permutation $\left(5^{*}\right)^{B_{i}}$ next. Since $B_{1} \cup B_{3}$ consists of all elements of order $2^{d},\left(5^{*}\right)^{B_{1}}$ and $\left(5^{*}\right)^{B_{3}}$ are $2^{d-2}$-cycles. As $B_{0}=\langle 4\rangle=Z_{2^{d-2}}$ and $B_{0} \cup B_{2}=\langle 2\rangle=$ $Z_{2^{d-1}}$, it is easy to deduce that $\left(5^{*}\right)^{B_{2}}$ is a product of two $2^{d-3}$-cycles (if $d=3$, then $\left(5^{*}\right)^{B_{2}}$ is trivial). Therefore the orders of $\left(5^{*}\right)^{B_{1}}$ and $\left(5^{*}\right)^{B_{3}}$ are $2^{d-2}$, the order of $\left(5^{*}\right)^{B_{2}}$ is $2^{d-3}$, and the order of $\left(5^{*}\right)^{B_{0}}$ is $2^{d-4}$ (if $d=3$, then the order is 1 ).

\section{Case 1: $d=3$}

In this case, $n=8$ and $\operatorname{Aut}\left(Z_{8}\right)=\left\langle(-1)^{*}\right\rangle \times\left\langle 5^{*}\right\rangle \cong Z_{2} \times Z_{2}$. By computation, $5^{*}$ fixes $B_{0}$ and $B_{2}$ pointwise, and the induced action $\left(5^{*}\right)^{B_{1}}=\hat{4}^{B_{1}}$ and $\left(5^{*}\right)^{B_{3}}=\hat{4}^{B_{3}}$. The element $(-1)^{*}$ fixes $B_{0}$ pointwise and $\left((-1)^{*}\right)^{B_{2}}=\hat{4}^{B_{2}}$.

Lemma 3.5. Let $\mathbb{Z}_{8}=\langle z\rangle$. Suppose that $G=\mathbb{Z}_{8} \rtimes G_{0}$ where $G_{0} \leqslant \operatorname{Aut}\left(\mathbb{Z}_{8}\right)=\left\langle(-1)^{*}\right\rangle \times$ $\left\langle 5^{*}\right\rangle$. Then the coset $z+Z_{2} \subseteq z^{G_{0}}$ if and only if $5^{*} \in G_{0}$ where $Z_{2}=\langle 4\rangle$ is the subgroup of order 2 .

Proof. Note that both $z$ and $z+Z_{2}$ are contained in $B_{1}$ or $B_{3}$ and $(-1)^{*}$ interchanges two blocks $B_{1}$ and $B_{3}$. The result follows from the analysis of the actions of $(-1)^{*}$ and $5^{*}$ easily.

Proposition 3.6. With above notation, let $G=Z_{8} \rtimes G_{0}$ where $G_{0} \leqslant \operatorname{Aut}\left(Z_{8}\right)=\left\langle(-1)^{*}\right\rangle \times$ $\left\langle 5^{*}\right\rangle$. Then

1. if $G_{0}=\operatorname{Aut}\left(Z_{8}\right)$ then $G$ is not 2-closed.

2. if $G_{0} \lesseqgtr \operatorname{Aut}\left(Z_{8}\right)$ and $G_{0} \neq\left\langle 5^{*}\right\rangle$, then $G$ is 2-closed and can be represented by an arc-transitive circulant.

3. if $G_{0}=\left\langle 5^{*}\right\rangle$, then $G$ is 2-closed and can be represented by a circulant. 
Proof. (1) Suppose first that $G_{0}=\operatorname{Aut}\left(Z_{8}\right)$. Let $\beta \in S_{8}$ such that $\beta$ fixes $B_{0}, B_{1}$ and $B_{3}$ pointwise and $\beta^{B_{2}}=\hat{4}^{B_{2}}$. Take any pair $\left(y_{1}, y_{2}\right) \in Z_{8} \times Z_{8}$. If both $y_{1}$ and $y_{2}$ belong to $B_{2}$, then $\left(y_{1}, y_{2}\right)^{\beta}=\left(y_{1}, y_{2}\right)^{\hat{4}}$ is in the orbital $\left(y_{1}, y_{2}\right)^{G}$. Suppose next that exactly one of $\left\{y_{1}, y_{2}\right\}$ belongs to $B_{2}$, say $y_{2} \in B_{2}$. It is straightforward to check that $\left(y_{1}, y_{2}\right)^{\beta}=\left(y_{1}, y_{2}\right)^{(-1)^{*}}$ if $y_{1} \in B_{0}$. Let $G_{1}$ be the point stabilizer of point 1 , then $G_{1}$ is the conjugate of $G_{0}$ by $\hat{1} \in \hat{\mathbb{Z}}_{n}$. Let $\alpha_{1}$ be the corresponding conjugate of $5^{*}$ in $G_{1}$. It follows that $\left(y_{1}, y_{2}\right)^{\beta}=\left(y_{1}, y_{2}\right)^{\alpha_{1}}$ if $y_{1} \in B_{1} \cup B_{3}$. Hence $\beta \in G^{(2)}$. However since $\beta$ fixes 0 and 1 , $\beta \notin G$ and so $G$ is not 2-closed.

(2) In this case, $5^{*} \notin G_{0}$. Let $S=1^{G_{0}}$ and let $\Gamma=\operatorname{Cay}\left(\mathbb{Z}_{8}, S\right)$. It follows from Lemma 3.5 and Theorem 2.1 that $G=\operatorname{Aut}(\Gamma)$ and is 2-closed.

(3) Finally we show that $\mathbb{Z}_{8} \rtimes\left\langle 5^{*}\right\rangle$ is 2-closed. Let $S_{1}=1^{\left\langle 5^{*}\right\rangle}=\{1,5\}$ and $S_{2}=2^{\left\langle 5^{*}\right\rangle}=$ $\{2\}$. Let $\Gamma=\operatorname{Cay}\left(\mathbb{Z}_{8}, S_{1} \cup S_{2}\right)$. By [12, Theorem 1.3], it is easy to deduce that $\Gamma$ is not arc-transitive. Suppose $g \in \operatorname{Aut}(\Gamma)$ such that $g$ fixes 0 and 1 , it is straightforward to check that $g=1$. We conclude that $\operatorname{Aut}(\Gamma)=\mathbb{Z}_{8} \rtimes\left\langle 5^{*}\right\rangle$ as required.

\section{Case 2: $d \geqslant 4$}

Let $\alpha=\left(5^{*}\right)^{2^{d-4}}$ be an element of order 4 in $\left\langle 5^{*}\right\rangle$. By the analysis of action of $5^{*}$, we deduce that $\alpha$ fixes $B_{0}$ pointwise and $o\left(\alpha^{B_{2}}\right)=2, o\left(\alpha^{B_{1}}\right)=o\left(\alpha^{B_{3}}\right)=4$.

Suppose first that $d=4$, then $\alpha=5^{*}$. By direct computation, $\alpha^{B_{2}}=\hat{8}^{B_{2}}, \alpha^{B_{1}}=\hat{4}^{B_{1}}$ and $\alpha^{B_{3}}=\widehat{-4}^{B_{3}}$.

Next suppose $d \geqslant 5$. Denote $N=\hat{\mathbb{Z}}_{n} \rtimes \mathbb{Z}_{n}^{*}$. Note that $N_{B_{i}}^{B_{i}} \cong \hat{Z}_{2^{d-2}} \rtimes K_{i}$ where $K_{i} \cong \operatorname{Aut}\left(Z_{2^{d-2}}\right)$ for each $i \in\{1,2,3\}$. Since $\left(5^{*}\right)^{B_{i}}$ is fixed point free on $B_{i}$ for $i=1,2,3$, $\left(5^{*}\right)^{B_{i}}=\hat{y}_{i}^{B_{i}} \tau_{i}$ where $0 \neq y_{i} \in \mathbb{Z}_{n}$ and $\tau_{i} \in K_{i}$. Since $\tau_{i}$ normalizes $\hat{Z}_{2^{d-2}},\left(\left(5^{*}\right)^{B_{i}}\right)^{2}=$ $\hat{y}_{i}^{B_{i}}\left(\tau_{i} \hat{y}_{i}^{B_{i}} \tau_{i}^{-1}\right) \tau_{i} \tau_{i}=a_{i 2} \tau_{i}^{2}$ where $a_{i 2}$ is some element in $\hat{Z}_{2^{d-2}}$. By induction, we have that for each $k \geqslant 1,\left(\left(5^{*}\right)^{B_{i}}\right)^{k}=a_{i k} \tau_{i}^{k}$ where $a_{i k}$ is some element in $\hat{Z}_{2^{d-2}}$. Since $\tau_{i} \in \operatorname{Aut}\left(Z_{2^{d-2}}\right)$ and $d \geqslant 5, \tau_{i}^{2^{d-4}}=1$. By the order of $\alpha^{B_{i}}$, we have that $\alpha^{B_{i}}=\hat{x}_{i}^{B_{i}}$, where $x_{1}, x_{3} \in Z_{n}$ are of order 4 and $x_{2}=2^{d-1}$ is the unique involution in $Z_{n}$. In addition, $2 x_{1}=2 x_{3}=2^{d-1}$. Therefore we have proved the following lemma.

Lemma 3.7. Suppose $d \geqslant 4$. With above notation, let $\alpha=\left(5^{*}\right)^{2^{d-4}}$ be an element of order 4 in $\left\langle 5^{*}\right\rangle$. Then $\alpha$ fixes $B_{0}$ pointwise, $\alpha^{B_{2}}=\left(\widehat{2^{d-1}}\right)^{B_{2}}, \alpha^{B_{1}}=\hat{x}_{1}^{B_{1}}$ for some $x_{1} \in Z_{n}$ with order 4 and $\alpha^{B_{3}}=\hat{x}_{3}^{B_{3}}$ for some $x_{3} \in Z_{n}$ with order 4 .

Corollary 3.8. Let $n=2^{d}$ for $d \geqslant 4$ and let $Z_{n}=\langle z\rangle$. Suppose that $G=Z_{n} \rtimes G_{0}$ where $G_{0} \leqslant \operatorname{Aut}\left(Z_{n}\right)=\left\langle(-1)^{*}\right\rangle \times\left\langle 5^{*}\right\rangle$. Let $\alpha \in\left\langle 5^{*}\right\rangle$ be of order 4 . Then

1. the coset $z+Z_{4} \subseteq z^{G_{0}}$ if and only if $\alpha \in G_{0}$ where $Z_{4} \leqslant Z_{n}$ is the subgroup of order 4.

2. the coset $z+Z_{2} \subseteq z^{G_{0}}$ if and only if $\alpha^{2} \in G_{0}$ where $Z_{2} \leqslant Z_{n}$ is the subgroup of order 2.

Proof. By Lemma 3.7, we have that $z+Z_{4} \subseteq z^{G_{0}}$ if $\alpha \in G_{0}$ and $z+Z_{2} \subseteq z^{G_{0}}$ if $\alpha^{2} \in G_{0}$.

With the notation in Lemma 3.7, suppose that $z+Z_{4} \subseteq z^{G_{0}}$. Note that $z \in B_{1}$ or $B_{3}$ and $z+Z_{4} \subseteq B_{1}$ or $B_{3}$ respectively. Since $(-1)^{*}$ interchanges $B_{1}$ and $B_{3}$, it is easy to deduce that $\alpha \in G_{0}$. Similarly, if $z+Z_{2} \subseteq z^{G_{0}}$ then $\alpha^{2} \in G_{0}$. 
Proposition 3.9. With above notation, let $G=Z_{n} \rtimes G_{0} \leqslant Z_{n} \rtimes \operatorname{Aut}\left(Z_{n}\right)$ where $n=2^{d}$ for $d \geqslant 4$. If $\alpha=\left(5^{*}\right)^{2^{d-4}} \in G_{0}$, then $\left(Z_{n} \rtimes\langle\alpha\rangle\right)^{(2)} \not \leq Z_{n} \rtimes \operatorname{Aut}\left(Z_{n}\right)$. In particular, $G$ is not 2-closed on $Z_{n}$.

Proof. Let $1 \neq \beta \in \operatorname{Sym}\left(Z_{2^{d}}\right)$ such that $\beta$ fixes $B_{0}, B_{2}, B_{3}$ pointwise and $\beta^{B_{1}}={\widehat{\left(2^{d-1}\right)}}^{B_{1}}$ is of order 2. Therefore $\beta^{B_{1}}=\left(\alpha^{2}\right)^{B_{1}}$. We will show next that $\beta \in\left(Z_{2^{d}} \rtimes\langle\alpha\rangle\right)^{(2)} \leqslant G^{(2)}$.

Take any pair $\left(y_{1}, y_{2}\right) \in Z_{n} \times Z_{n}$. If both $y_{1}$ and $y_{2}$ belong to $B_{1}$, then $\left(y_{1}, y_{2}\right)^{\beta}=$ $\left(y_{1}, y_{2}\right)^{\widehat{2^{d-1}}}$ is in the orbital $\left(y_{1}, y_{2}\right)^{G}$. Suppose next that exactly one of $\left\{y_{1}, y_{2}\right\}$ belongs to $B_{1}$, say $y_{2} \in B_{1}$. By Lemma 3.7, $\left(y_{1}, y_{2}\right)^{\beta}=\left(y_{1}, y_{2}\right)^{\alpha^{2}}$ if $y_{1} \in B_{0}$ or $B_{2}$. Let $G_{3}$ be the point stabilizer of point 3 , then $G_{3}$ is the conjugate of $G_{0}$ by $\hat{3} \in \hat{Z}_{n}$. Let $\alpha_{3}$ be the corresponding conjugate of $\alpha$ in $G_{3}$, it follows from Lemma 3.7 that $\left(y_{1}, y_{2}\right)^{\beta}=\left(y_{1}, y_{2}\right)^{\alpha_{3}}$ if $y_{1} \in B_{3}$. Thus $\beta \in\left(Z_{2^{d}} \rtimes\langle\alpha\rangle\right)^{(2)} \leqslant G^{(2)}$. However since $\beta$ fixes $B_{0}$ and $B_{3}$ pointwise, $\beta \notin Z_{2^{d}} \rtimes \operatorname{Aut}\left(Z_{2^{d}}\right)$ and so $\left(Z_{2^{d}} \rtimes\langle\alpha\rangle\right)^{(2)} \not \leq Z_{2^{d}} \rtimes \operatorname{Aut}\left(Z_{2^{d}}\right)$. In particular $G$ is not 2-closed.

Next we will show that if $\alpha \notin G_{0}$ then $G$ is 2 -closed. Note that $\alpha \notin G_{0}$ is equivalent to the condition that either $\left|G_{0}\right| \leqslant 2$ or $\left|G_{0}\right|=4$ and $G_{0} \nless\left\langle 5^{*}\right\rangle$.

We first discuss the case that $\alpha^{2} \notin G_{0}$.

Lemma 3.10. With above notation, let $n=2^{d}$ for $d \geqslant 4$. Let $G=Z_{n} \rtimes G_{0}$. Suppose $\alpha^{2} \notin G_{0}$. Then $G$ is the full automorphism group of an arc-transitive circulant and so $G$ is 2-closed.

Proof. Let $S=1^{G_{0}}$ be the orbit of 1 under $G_{0}$, and let $\Gamma=\operatorname{Cay}\left(Z_{n}, S\right)$. Since $\alpha^{2} \notin G_{0}$, it follows from corollary 3.8 that $S$ is not a union of cosets of any subgroup of $Z_{n}$. By Theorem 2.1, $\operatorname{Aut}(\Gamma)=G$ as required.

It remains to show that if $G=Z_{n} \rtimes G_{0}$ where $\alpha^{2} \in G_{0}$ but $\alpha \notin G_{0}$ then $G$ is the full automorphism group of some circulant. We will prove this in Proposition 3.15 when we handle the more general case.

\subsection{The general case.}

\subsubsection{The notation for the main theorem.}

We explain Conditions 1.1 in more detail first.

Let

$$
n=2^{d_{1}} p_{2}^{d_{2}} \cdots p_{t}^{d_{t}}, \quad d_{1} \geqslant 0, d_{2}, \ldots, d_{t} \geqslant 1, t \geqslant 1
$$

where $p_{2}, \ldots, p_{t}$ are distinct odd primes. For convenience, we also write $p_{1}=2$. In addition, the notion $p_{i}^{d_{i}}|| n$ means $p_{i}^{d_{i}} \mid n$ but $p_{i}^{d_{i}+1} \nmid n$.

Let $G=\hat{Z}_{n} \rtimes G_{0}$ acting on $Z_{n}$ naturally where $G_{0} \leqslant \operatorname{Aut}\left(Z_{n}\right)$. In order to reduce the proof in the general case to the prime power case, we choose the product action form to describe $G$. Let $Z_{m}$ be the unique subgroup of $Z_{n}$ of order $m$ for $m \mid n$. Then we may write

$$
Z_{n}=Z_{2^{d_{1}}} \times Z_{p_{2}^{d_{2}}} \times \cdots \times Z_{p_{t}^{d_{t}}}=\left\{\left(z_{1}, \ldots, z_{t}\right)=z_{1} z_{2} \cdots z_{t} \mid z_{i} \in Z_{p_{i}^{d_{i}}}, \text { where } p_{1}=2\right\} .
$$


For any $g=\left(g_{1}, \ldots, g_{t}\right) \in Z_{n}$, we have $\hat{g}:\left(z_{1}, \ldots, z_{t}\right) \mapsto\left(z_{1} g_{1}, \ldots, z_{t} g_{t}\right)$. Moreover,

$$
\operatorname{Aut}\left(Z_{n}\right)=\operatorname{Aut}\left(Z_{2^{d_{1}}}\right) \times \cdots \times \operatorname{Aut}\left(Z_{p_{t}^{d_{t}}}\right)=D_{1} D_{2} \cdots D_{t}
$$

where $D_{i}$ is the direct factor subgroup of $\operatorname{Aut}\left(Z_{n}\right)$ that fixes each component of the elements of $Z_{n}$ except for the $i$-th component. So $D_{i} \cong \operatorname{Aut}\left(Z_{p_{i}}\right)$.

In fact $D_{i}$ induces a faithful action on the subgroup $Z_{p_{i}}^{d_{i}}$. With notation in $\S 3.2$, if $d_{1} \geqslant 3$ then the induced action $D_{1}$ on the subgroup $Z_{2^{d_{1}}}$ is permutation isomorphic to $\left\langle(-1)^{*}\right\rangle \times\left\langle 5^{*}\right\rangle\left(d_{1} \geqslant 3\right)$, the multiplicative group of units of the ring $\mathbb{Z}_{2^{d_{1}}}$ acting on the additive group $\mathbb{Z}_{2^{d_{1}}}$. Let $\phi:\left\langle(-1)^{*}\right\rangle \times\left\langle(5)^{*}\right\rangle \rightarrow D_{1}$ be the corresponding group isomorphism.

The normalizer of $\hat{Z}_{n}$ in $\operatorname{Sym}\left(Z_{n}\right)$ is

$$
N=\hat{Z}_{n} \rtimes \operatorname{Aut}\left(Z_{n}\right)=\left(\hat{Z}_{2^{d_{1}}} \rtimes \operatorname{Aut}\left(Z_{2^{d_{1}}}\right)\right) \times \cdots \times\left(\hat{Z}_{p_{t}^{d_{t}}} \rtimes \operatorname{Aut}\left(Z_{p_{t}^{d_{t}}}\right)\right)
$$

acting on $Z_{n}$ by the natural product action. Therefore $G=\hat{Z}_{n} \rtimes G_{0} \leqslant N$ has the natural product action.

We need the following two easy observations in the proof below.

(1) Note that when $i \geqslant 2, \operatorname{Aut}\left(Z_{p_{i}}\right)=Z_{p-1} \times Z_{p_{i}^{d_{i}-1}}$. Conditions 1.1 [ii] is equivalent to $\alpha_{i} \notin G_{0}$ where $\alpha_{i} \in D_{i} \cong Z_{p_{i}-1} \times Z_{p_{i}^{d_{i}-1}}$ is of order $p_{i}$.

(2) When $i=1$ and $d_{1} \geqslant 4$, denote $\alpha_{1}=\phi\left(\left(5^{*}\right)^{2^{d_{1}-4}}\right) \in D_{1}$, then the order of $\alpha_{1}$ is 4 . Conditions 1.1 [iv] is equivalent to $\alpha_{1} \notin G_{0}$.

\subsubsection{The proof of Theorem 1.2.}

Lemma 3.11. With notation in Subsection 3.3.1, suppose $G=\hat{Z}_{n} \rtimes G_{0}$ where $G_{0} \leqslant$ $\operatorname{Aut}\left(Z_{n}\right)$. If $G_{0}$ fails to satisfy one of conditions 1.1, then $G$ is not 2-closed.

Proof. If condition (i) does not hold, then there exists an odd prime $p_{i} \geqslant 5$ where $i \geqslant 2$ such that $p_{i} \| n$ and $D_{i} \leqslant G_{0}$. In this case we take $K=\hat{Z}_{p_{i}} \rtimes D_{i}$. By hypothesis, $K$ is the subgroup of $G$ which fixes each component of elements of $Z_{n}$ except for the $i$-th component. Hence the action of $K$ on $Z_{n}$ is the product action of $\bar{K} \times\{1\}$ on $Z_{n}=Z_{p_{i}} \times Z_{\frac{n}{p_{i}}}$ where $\bar{K} \cong K$ acts on $Z_{p_{i}}$ naturally. It follows from Theorem 2.3 that $K^{(2)}=(\bar{K})^{(2)} \times\{1\}$. By the remark after Corollary $2.2,(\bar{K})^{(2)} \nsubseteq Z_{p_{i}} \rtimes \operatorname{Aut}\left(Z_{p_{i}}\right)$. Since $G^{(2)} \geqslant K^{(2)}$, we have that $G$ is not 2-closed in this case.

If condition (ii) does not hold, then there exists an odd prime $p_{i}$ where $i \geqslant 2$ such that $p_{i}^{d_{i}} \| n$ and $d_{i} \geqslant 2$. Since $\alpha_{i} \in G_{0}$ in this case, we take $K=\hat{Z}_{p_{i}^{d_{i}}} \rtimes\left\langle\alpha_{i}\right\rangle \leqslant G$. Hence the action of $K$ on $Z_{n}$ is the product action of $\bar{K} \times\{1\}$ on $Z_{n}=Z_{p_{i}^{d_{i}}} \times Z_{\frac{n}{p_{i}}}$ where $\bar{K} \cong K$ acts on $Z_{p_{i}^{d_{i}}}$ naturally. By the remark after Proposition 3.3, $(\bar{K})^{(2)} \nsubseteq Z_{p_{i}^{d_{i}}} \rtimes \operatorname{Aut}\left(Z_{p_{i}^{d_{i}}}\right)$. The same argument as above proves that $G$ is not 2-closed in this case either.

Suppose $2^{d_{1}} \| n$ and $d_{1} \geqslant 3$, suppose also that either condition (iii) or (iv) fails. Take $K=\widehat{Z_{8}} \rtimes D_{1}$ if $d_{1}=3$ and take $K=\widehat{Z_{2^{d_{1}}}} \rtimes\left\langle\alpha_{1}\right\rangle$ if $d_{1} \geqslant 4$. By the same argument as above, it follows from Proposition 3.6(1) and Proposition 3.9 that $G$ is not 2-closed. 
Lemma 3.12. With notation in Subsection 3.3.1, suppose $G=\hat{Z}_{n} \rtimes G_{0}$ where $G_{0} \leqslant$ $\operatorname{Aut}\left(Z_{n}\right)$ and $G_{0}$ satisfies Conditions 1.1. Let $S=z^{G_{0}}$ where $Z_{n}=\langle z\rangle$, and let $\Gamma=$ Cay $\left(Z_{n}, S\right)$. Then exactly one of the following holds.

1. $G$ is the full automorphism group of $\Gamma$ and so $G$ is 2-closed and can be represented by an arc-transitive circulant.

2. $2^{d_{1}} \| n, d_{1} \geqslant 4$, and $\alpha_{1}^{2} \in G_{0} \cap D_{1}$.

3. $2^{3} \| n$, and $D_{1} \cap G_{0}=\left\langle\phi\left(5^{*}\right)\right\rangle \cong Z_{2}$.

4. $n=4 m$ where $m>1$ is odd. $D_{1} \cap G_{0}=D_{1} \cong Z_{2}$, that is $G_{0}=\operatorname{Aut}\left(Z_{4}\right) \times K$ where $K \leqslant \operatorname{Aut}\left(Z_{m}\right)$.

Moreover, in the latter three cases, $\Gamma=\Sigma\left[\bar{K}_{2}\right]$ is a lexicographic product and the pointwise stabilizer of $\{1, z\}$ in $\operatorname{Aut}(\Gamma)$ preserves each coset of $Z_{2}$.

Proof. Suppose that $G$ is not the full automorphism group of $\Gamma$. By the condition (i), for any odd prime $p_{i} \geqslant 5$ such that $p_{i} \| n$, we have $G_{0} \neq \operatorname{Aut}\left(Z_{p_{i}}\right) \times H$ for some $H \leqslant$ $\operatorname{Aut}\left(Z_{n / p_{i}}\right)$. It then follows from Theorem 2.1 that case (b) of Theorem 2.1 occurs for $\Gamma$. That is $n=b k>4$ where $b \geqslant 2$ and $\Gamma=\Sigma\left[\bar{K}_{b}\right]$. Moreover, the group $Z_{n}$ has a subgroup $Y$ of order $b$ and $S$ is a union of $Y$-cosets each consisting of generators for $Z_{n}$.

Recall that $n=2^{d_{1}} p_{2}^{d_{2}} \cdots p_{t}^{d_{t}}$. Suppose that $p_{j} \mid b$ for some $j \in\{1, \ldots, t\}$. Then $z Z_{p_{j}} \subseteq S$ where $Z_{p_{j}}$ is the subgroup of order $p_{j}$ and $d_{j} \geqslant 2$ by Theorem 2.1 (b). Let $z=\left(z_{1}, \ldots, z_{t}\right)$ where $z_{i}$ is a generator of $Z_{p_{i} d_{i}}$ for each $i$. Thus $z Z_{p_{j}} \subseteq S=z^{G_{0}}$ implies that $z_{j} Z_{p_{j}} \subseteq z_{j}^{D_{j} \cap G_{0}}$ in the $j$-th component. By Corollary 3.2, the condition (ii) implies that $b=2^{l}$ is a power of 2. Similarly, by Corollary 3.8, Lemma 3.5 and the action of Aut $\left(Z_{4}\right)$, the condition (iii) and (iv) imply that $b$ must be 2 and one of cases $2-4$ happens.

Suppose next that one of cases $2-4$ occurs. Thus $\Gamma=\Sigma\left[\bar{K}_{2}\right]$ where $\Sigma=\operatorname{Cay}\left(Z_{n} / Z_{2}, \bar{S}\right)$ and $\bar{S}=\left\{s Z_{2} \mid s \in S\right\}$. Moreover, by [14, Lemma 2.3], the set $\left\{x Z_{2} \mid x \in Z_{n}\right\}$ forms a block system of $\operatorname{Aut}(\Gamma)$, and so $\operatorname{Aut}(\Gamma)=Z_{2} \prec \operatorname{Aut}(\Sigma)$.

Let $\bar{G}_{0}=G_{0} /\left\langle\alpha_{1}^{2}\right\rangle$ in case 2 , and let $\bar{G}_{0}=G_{0} /\left(D_{1} \cap G_{0}\right)$ in case 3 or 4 . Then $\bar{G}_{0} \leqslant \operatorname{Aut}\left(Z_{n} / Z_{2}\right)$ and $\bar{S}=\left(z Z_{2}\right)^{\bar{G}_{0}}$. Note that $G_{0}$ satisfies Conditions 1.1, it follows that $\bar{S}$ is not the union of cosets of any subgroup of $Z_{n} / Z_{2}$. By Theorem 2.1, $\Sigma$ is normal and $\operatorname{Aut}(\Sigma)=\left(Z_{n} / Z_{2}\right) \rtimes \bar{G}_{0}$. Therefore the pointwise stabilizer of $\{1, z\}$ in $\operatorname{Aut}(\Gamma)$ preserves each coset of $Z_{2}$.

Remark: Suppose $G$ satisfies Conditions 1.1. By the above lemma, $G$ can be represented by an arc-transitive circulant if and only if $G$ does not arise in any of the cases 2-4 of Lemma 3.12.

Next we will show that if one of cases 2-4 occurs then there exists a circulant $\Gamma$ which is not arc-transitive such that $\operatorname{Aut}(\Gamma)=G$. We discuss case 4 first.

Lemma 3.13. Suppose $n=4 m$ where $m>1$ is odd and $G=Z_{4 m} \rtimes G_{0}$ where $G_{0}=$ $\operatorname{Aut}\left(Z_{4}\right) \times K$ and $K \leqslant \operatorname{Aut}\left(Z_{m}\right)$. Suppose further that $G_{0}$ satisfies Conditions 1.1. Then $G$ is 2 -closed and can be represented by a circulant. 
Proof. Let $z=z_{1} z_{2} \in Z_{4 m}$ where $z_{1}$ is a generator of $Z_{4}$ and $z_{2}$ is a generator of $Z_{m}$. Let $S_{1}=z^{G_{0}}$ and $\Gamma_{1}=\operatorname{Cay}\left(Z_{4 m}, S_{1}\right)$. By Lemma 3.12, $S_{1}$ is the union of some cosets of $Z_{2}=$ $\left\langle z_{1}^{2}\right\rangle$. Let $S_{2}=z_{2}^{G_{0}} \subseteq Z_{m}$ and $\Gamma_{2}=\operatorname{Cay}\left(Z_{4 m}, S_{2}\right)$. Thus $B_{0}=Z_{m}, B_{1}=z Z_{m}, B_{2}=z^{2} Z_{m}$ and $B_{3}=z^{3} Z_{m}$ are the connected components of $\Gamma_{2}$.

Let $S=S_{1} \cup S_{2}$ and $\Gamma=\operatorname{Cay}\left(Z_{4 m}, S\right)$. Suppose first that $\Gamma$ is arc-transitive. Note that $S_{1}$ consists of elements of order $4 m$ and $S_{2}$ contains elements of order $m$. We observe that $S$ is not the union of cosets of any subgroup. By [12, Theorem 1.3], $\Gamma=\Sigma\left[\bar{K}_{b}\right]-b . \Sigma$ where $n=b r, 4 \leqslant b<n$ and $\operatorname{gcd}(b, r)=1$. Thus writing $Z_{n}=Y \times M$ with $Y \cong Z_{b}$ and $M \cong Z_{r}$, we have that $S=Y \backslash\{1\} \times T$ and $T \subseteq M \backslash\{1\}$. Analyzing the orders of elements of $S$, we have that $b=p_{i}$ is prime, $p_{i} \geqslant 5$ and $p_{i} \| m$ as $(b, r)=1$. As $z^{G_{0}} \subset Y \backslash\{1\} \times T$, $D_{i} \cong \operatorname{Aut}\left(Z_{p_{i}}\right) \subseteq G_{0}$, contradicting the condition (i). Thus $\Gamma$ is not arc-transitive.

Let $P$ be the point stabilizer of $\operatorname{Aut}(\Gamma)$ on vertex 1 . Since $P \geqslant G_{0}, P$ has two orbits $S_{1}$ and $S_{2}$ and so $\operatorname{Aut}(\Gamma)=\operatorname{Aut}\left(\Gamma_{1}\right) \cap \operatorname{Aut}\left(\Gamma_{2}\right)$

Assume that $g \in \operatorname{Aut}(\Gamma)$ fixing $1 \in B_{0}$ and $z \in B_{1}$. Consider $z^{2} \in B_{2} \cap z S_{1}$ which is adjacent to $z$. It follows from Lemma 3.12 that $g$ fixes each coset of $Z_{2}=\left\langle z_{1}^{2}\right\rangle$. Hence $\left(z^{2}\right)^{g} \in\left\{z^{2}, z^{2} z_{1}^{2}\right\}=z^{2} Z_{2}$ and $g$ fixes both $z \in B_{1}$ and $z z_{1}^{2} \in B_{3}$. Moreover, as $g \in \operatorname{Aut}\left(\Gamma_{2}\right)$, we conclude that $g$ must fix $B_{0}, B_{1}, B_{2}$ and $B_{3}$ setwise. Therefore, $g$ fixes $z^{2}$. Continuing in this fashion, we conclude that $g$ fixes $z^{3}, z^{4}, \ldots$ and so on. Thus $g=1$ and $P=G_{0}$. It follows that $\operatorname{Aut}(\Gamma)=G$ as required.

It remains to handle case 2 and case 3 in Lemma 3.12. By Lemma 3.12, we may suppose that $8 \mid n$ and $G=\hat{Z}_{n} \rtimes G_{0}$ where $G_{0} \leqslant \operatorname{Aut}\left(Z_{n}\right)$. Let $S_{1}=z^{G_{0}}$ where $Z_{n}=\langle z\rangle$ and let $S_{2}=\left(z^{2}\right)^{G_{0}} \subseteq Z_{n / 2}=\left\langle z^{2}\right\rangle$. We construct $\Gamma=\operatorname{Cay}\left(Z_{n}, S_{1} \cup S_{2}\right)$. We will show that $\Gamma$ can represent $G$ in both case 2 and case 3 . In order for proving this, let $\Gamma_{1}=\operatorname{Cay}\left(Z_{n}, S_{1}\right)$ and $\Gamma_{2}=\operatorname{Cay}\left(Z_{n}, S_{2}\right)$ we need to study $\Gamma_{1}$ and $\Gamma_{2}$. Note that $\Gamma_{1}$ has been studied in Lemma 3.12. We study $\Gamma_{2}$ in the following lemma.

Lemma 3.14. Suppose that case 2 or 3 of Lemma 3.12 occurs. With above notation, we have that $\Gamma_{2}=2 \cdot \operatorname{Cay}\left(\left\langle z^{2}\right\rangle, S_{2}\right)$. Let $A_{3}=\operatorname{Aut}\left(\operatorname{Cay}\left(\left\langle z^{2}\right\rangle, S_{2}\right)\right)$ and $A_{2}=\operatorname{Aut}\left(\Gamma_{2}\right)$. Then $\left.A_{2}=A_{3}\right\rangle Z_{2}$. Moreover, $\operatorname{Cay}\left(\left\langle z^{2}\right\rangle, S_{2}\right)$ is a normal arc-transitive circulant and $A_{3}=\left\langle z^{2}\right\rangle \rtimes G_{0}^{\left\langle z^{2}\right\rangle}$.

Proof. Let $\Delta_{1}=\left\langle z^{2}\right\rangle$ and $\Delta_{2}=z\left\langle z^{2}\right\rangle$. Then $\Gamma_{2}=2$. Cay $\left(\left\langle z^{2}\right\rangle, S_{2}\right)$ such that $\Delta_{1}$ and $\Delta_{2}$ are two connected components of $\Gamma_{2}$. Thus $A_{2}=A_{3} \zeta Z_{2}$.

Let $\bar{G}_{0}=G_{0} /\left\langle\alpha_{1}^{2}\right\rangle$ in case 2 , and let $\bar{G}_{0}=G_{0} /\left(D_{1} \cap G_{0}\right)$ in case 3 . Note that $G_{0}$ preserves $\Delta_{1}$, it is easy to check that the induced permutation group $G_{0}^{\Delta_{1}} \cong \bar{G}_{0}$ and $G_{0}^{\Delta_{1}} \leqslant \operatorname{Aut}\left(\left\langle z^{2}\right\rangle\right)$. Also $S_{2}=\left(z^{2}\right)^{G_{0}^{\Delta_{1}}}$ is an orbit of $G_{0}^{\Delta_{1}}$. Since $G_{0}$ satisfies conditions in Theorem 1.2, $S_{2}$ is not the union of cosets of any subgroup of $\left\langle z^{2}\right\rangle$. By Theorem 2.1 and Conditions 1.1, we conclude that $\operatorname{Cay}\left(\left\langle z^{2}\right\rangle, S_{2}\right)$ is normal and $\operatorname{Aut}\left(\operatorname{Cay}\left(\left\langle z^{2}\right\rangle, S_{2}\right)\right)=$ $\left\langle z^{2}\right\rangle \rtimes G_{0}^{\Delta_{1}}$.

Proposition 3.15. With notation in Subsection 3.3.1, suppose $G=\hat{Z}_{n} \rtimes G_{0}$ where $G_{0} \leqslant \operatorname{Aut}\left(Z_{n}\right)$ and $G_{0}$ satisfies Conditions 1.1. Suppose further that case 2 or 3 of Lemma 3.12 occurs. Let $S_{1}=z^{G_{0}}$ where $Z_{n}=\langle z\rangle$ and let $S_{2}=\left(z^{2}\right)^{G_{0}}$. Let $\Gamma=\operatorname{Cay}\left(Z_{n}, S_{1} \cup S_{2}\right)$ and let $P$ be the point stabilizer of vertex 1 in $\operatorname{Aut}(\Gamma)$. Then 
1. $\Gamma$ is not arc-transitive, and $S_{1}, S_{2}$ are two orbits of $P$.

2. For any $g \in \operatorname{Aut}(\Gamma)$ such that $g$ fixes 1 and $z$, we have that $g=1$.

3. $\operatorname{Aut}(\Gamma)=G=Z_{n} \rtimes G_{0}$. So $\Gamma$ is normal and $G$ is 2-closed.

Proof. (1) Suppose, to the contrary, that $\Gamma$ is arc-transitive. Note that $S_{1}$ consists of elements of order $n$ and $S_{2}$ contains elements of order $n / 2 \neq n$. Also observe that $S$ is not the union of cosets of any subgroup. By [12, Theorem 1.3], $\Gamma=\Sigma\left[\bar{K}_{b}\right]-b . \Sigma$, where $n=b r, 4 \leqslant b<n$ and $\operatorname{gcd}(b, r)=1$. Thus writing $Z_{n}=Y \times M$ with $Y \cong Z_{b}$ and $M \cong Z_{r}$, we have that $S=Y \backslash\{1\} \times T$ and $T \subseteq M \backslash\{1\}$. Analyzing the orders of elements of $S$, by conditions (i) (ii) we have that $b=4$. As $(b, r)=1,4 \| n$, contradicting the fact that $8 \mid n$. Thus $\Gamma$ is not arc-transitive. As $P \geqslant G_{0}, S_{1}, S_{2}$ are two orbits of $P$.

(2) Let $\Gamma_{1}=\operatorname{Cay}\left(Z_{n}, S_{1}\right), \Gamma_{2}=\operatorname{Cay}\left(Z_{n}, S_{2}\right)$ and $A_{1}=\operatorname{Aut}\left(\Gamma_{1}\right), A_{2}=\operatorname{Aut}\left(\Gamma_{2}\right)$. It follows from (1) that $\operatorname{Aut}(\Gamma)=A_{1} \cap A_{2}$.

Let $g \in \operatorname{Aut}(\Gamma)$ such that $g$ fixes 1 and $z$. By Lemma 3.12, $g$ preserves each coset of $Z_{2}$ and so $\left(z^{2}\right)^{g} \in\left\{z^{2}, z^{2} z^{n / 2}\right\}$. Moreover, since $z^{2} \in S_{2}$ and $g$ preserves $S_{2}$, we have $\left(z^{2}\right)^{g} \in S_{2}$. By the proof of Lemma 3.14, we have that $z^{2} Z_{2} \nsubseteq S_{2}$ and so $z^{2} z^{n / 2} \notin S_{2}$. Thus $g$ fixes $z^{2}$. Let $\Delta_{1}=\left\langle z^{2}\right\rangle$ and $\Delta_{2}=z\left\langle z^{2}\right\rangle$ be two connected components of $\Gamma_{2}$. By Lemma 3.14, $g^{\Delta_{1}} \in \operatorname{Aut}\left(\left\langle z^{2}\right\rangle\right)$ fixes $\Delta_{1}$ pointwise. Now $g$ fixes $z$ and $z^{2}$ and consider $\left(z^{3}\right)^{g}$. Using the same argument we deduce that $g$ fixes $\Delta_{2}$ pointwise and so $g=1$.

(3) It follows from (2) that $P=G_{0}$ and so $A=G=\hat{Z}_{n} \rtimes G_{0}$. Therefore $\Gamma$ is normal and $G$ is 2-closed on $Z_{n}$.

Theorem 1.2 now follows from Lemma 3.11, Lemma 3.12, Lemma 3.13 and Proposition 3.15 .

\section{References}

[1] B. Alspach, On constructing the graphs with a given permutation group as their group, Proc. Fifth Southeastern Conf. Combin., Graph Theory and Computing (1974), 187-208.

[2] L. Babai, Finite digraphs with given regular automorphism groups, Periodica Mathematica Hungarica Vol. 11 (4), (1980), 257-270.

[3] L. Babai and C.D. Godsil, On the automorphism groups of almost all Cayley graphs, Europ. J. Combin. 3 (1982) 9-15.

[4] L. Babai, Automorphism groups, Isomorphism, Reconstruction, Chapter 27 of the Handbook of Combinatorics, 1447-1540, Edited by R. L. Graham, M. Grotschel and L. Lovasz, North-Holland, 1995.

[5] P. J. Cameron, M. Giudici, G. A. Jones, W. M. Kantor, M. H. Klin, D. Marušič, and L. A. Nowitz, Transitive permutation groups without semiregular subgroups, $J$. London Math. Soc. 66 (2002), 325-333. 
[6] E.Dobson, P.Spiga and G. Verret, Cayley graphs on abelian groups, Combinatorica (2014) in press.

[7] S.A. Evdokimov and I.N. Ponomarenko, Characterization of cyclotomic shcems and normal Schur rings over a cyclic group, (Russian), Algebra i Analiz 14(2) (2002),1155 .

[8] C.D. Godsil, GRR's for non-solvable groups, Algebraic Methods in Graph Theory, Colloq. Math. Soc. J. Bolyai, 25. Szeged, 1978; North-Holland, Amsterdam, 1981; 221-239.

[9] C.D. Godsil, Neighbourhoods of transitive graphs and GRR's, J. Combin. Theory Ser. B 29 (1980), 116-140.

[10] C.D. Godsil, On the full automorphism group of a graph, Combinatorica 1 (1981), 243-256.

[11] I. Kovács, Classifying arc-transitive circulants, J. Algebraic Combin. 20 (2004), 353-358.

[12] C. H. Li, Permutation groups with a cyclic regular subgroup and arc transitive circulants, J. Algebraic Combin. 21 (2005), 131-136.

[13] J.Morris, P.Spiga and G.Verret, Automorphisms of Cayley graphs on generalised dicyclic groups, Europ. J. Combin. 43 (2015) 68-81.

[14] C. E. Praeger and J. Xu, A note on arc-transitive circulant digraphs, J. Group Theory 12 (2009) 107-117.

[15] H. Wielandt, Permutation Groups Through Invariant Relations and Invariant Functions, Lecture Notes, Ohio State University, Columbus, 1969. Also publised in Wielandt, Helmut, Mathematische Werke/Mathematical works. Vol. 1. Group theory. Walter de Gruyter \& Co., Berlin, 1994, pp.237-296.

[16] Jing $\mathrm{Xu}$, Vertex-transitive tournaments of order a product of two distinct primes, $J$. Group Theory, 13 (2010), 565-576.

[17] Jing Xu, Metacirculant tournaments whose order is a product of two distinct primes, Discrete Mathematics 311 (2011) 571-576.

[18] Ming-Yao Xu, Automorphism groups and isomorphisms of Cayley digraphs, Discrete Math., 182 (1998), 309-319. 